\title{
A new implicit iteration process for two strongly pseudocontractive mappings
}

\author{
ARIF RAFIQ and ANA MARIA ACU
}

\section{ABSTRACT.}

The purpose of this paper is to establish the strong convergence of an implicit iteration process to a common fixed point for two continuous strongly pseudocontractive mappings.

\author{
HAJVERY UNIVERSITY \\ 43-52 Industrial ARea, GUlberg-III LAHORE, PAKISTAN \\ E-mail address: aarafiqegmail.com
}

UNIVERSITY "LUCIAN BLAGA" OF SiBIU

DEPARTMENT OF MATHEMATICS AND INFORMATICS

DR. I. RAṬIU 5-7, 550012 - SIBIU, ROMANIA

E-mail address: acuana77@yahoo.com

Received: 14.09.2010; In revised form: 16.03.2012; Accepted: 31.07.2012

2010 Mathematics Subject Classification. 47H10, 47H17, 54H25.

Key words and phrases. Implicit iteration process, strongly pseudocontractive mappings, L-Lipschitzian mappings, strong convergence, common fixed point.

Corresponding author: Ana Maria Acu; acuana77@yahoo.com 